\title{
Persicaria senegalensis (Polygonaceae), entità nuova per la flora italiana, e chiave di identificazione delle specie del genere Persicaria in Italia
}

\author{
Gabriele Galasso $^{1}$, Erina Montoleone ${ }^{2}$, Carmelo Federico ${ }^{3}$
}

${ }^{1}$ Corresponding author: Gabriele Galasso, Sezione di Botanica, Museo di Storia Naturale di Milano, Corso Venezia 55, 20121 Milano, Italia. E-mail: gabriele.galasso@comune.milano.it

2 Via Mainetti 28, 25136 Brescia, Italia. E-mail: persicaria.attenuata@gmail.com

${ }^{3}$ Viale Teocrito 1, 90135 Palermo, Italia. E-mail: hormuzakia@libero.it

\begin{abstract}
Riassunto - Viene riferito il ritrovamento a Pantelleria di Persicaria senegalensis, specie sinora mai segnalata in Italia. Segue una descrizione dettagliata e una chiave di tutte le specie del genere Persicaria, autoctone ed esotiche, presenti in Italia.
\end{abstract}

\footnotetext{
Abstract - Persicaria senegalensis (Polygonaceae), new for Italian flora and a key for Persicaria species in Italy.

The discovery on Pantelleria of Persicaria senegalensis, a species previously never recorded in Italy, is reported. A detailed description and a key to all the Italian species of the genus Persicaria, natives and aliens, is provided.
}

Key-words: Italian flora, Pantelleria, Persicaria senegalensis.

\section{INTRODUZIONE}

Il genere Persicaria (L.) Mill. appartiene alla famiglia delle Polygonaceae ed è uno dei numerosi generi derivati dalla suddivisione di Polygonum L. Esso è stato recentemente ridelimitato da Galasso et al. (2009) e comprende circa 100 specie, delle quali 16 presenti in Italia. Il numero delle specie italiane è andato crescendo, soprattutto in quest'ultimo decennio, arricchendosi di entità esotiche. Pignatti (1982) ne riporta soltanto 9, mentre Conti et al. (2005) ne aggiunge altre 3: P. capitata in Piemonte, segnalata sulla base di osservazioni a Cannobio (VB) di Bruno Cerabolini, $P$. pensylvanica in Lombardia (Brusa \& Galasso, 2006) e $P$. virginiana in Lombardia (Galasso et al., 2006). L'anno successivo sono state segnalate $P$. filiformis in Lombardia (Galasso \& Brusa, 2007), P.bungeana in Veneto (Galasso \& Tomasi (2007) e P. longiseta in Lombardia (Galasso, 2007).

Nell'anno 2000 sull'isola di Pantelleria (Sicilia, provincia di Trapani), presso la località L'Arenella, veniva rinvenuta una nuova specie di Persicaria diverse da tutte quelle sinora conosciute in Italia, molto alta e caratterizzata dall'essere ricoperta da una fitta lanosità biancastra, qui identificata come $P$. senegalensis. L'Arenella è l'unico tratto di costa pianeggiante e con accumuli di sabbia distribuiti all'interno delle depressioni rocciose. Qui è presente una grossa vasca, quasi certa- mente una vecchia cisterna, contenete acqua stagnante (coord. geogr. WGS84: $36.826928^{\circ} \mathrm{N} 11.932977^{\circ} \mathrm{E}$ ) e sulle cui rive non può passare inosservata $P$. senegalensis (Figg. 1-2).

Non è escluso che in futuro altre entità, originarie dell'Asia orientale e recentemente segnalate negli Stati Uniti d'America in climi analoghi a quelli delle regioni settentrionali, possano giungere anche in Italia; tra queste $P$. extremiorientalis (Atha et al., 2010) e P. perfoliata (Poindexter, 2010).

\section{MATERIALI E METODI}

Sono stati raccolti diversi campioni per la preparazione degli exsiccata, poi depositati in MSNM, ed effettuati confronti morfologici utilizzando le varie chiavi analitiche e le descrizioni a disposizione (Graham, 1958; Zohary, 1966; Akeroyd, 1988; Wilson, 1990; Thulin, 1993; Nyberg \& Miller, 1996; Boulos, 1999; El Husseini \& El Hadidi, 2000).

\section{RISULTATI E DISCUSSIONE}

L'identificazione delle piante di Pantelleria si è rivelata un po' difficoltosa in quanto le ocree presentano fimbrie molto evidenti, carattere non riportato dalla maggior parte degli autori per Persicaria senegalensis (es. Wilson, 1990), ma che in realtà possono giungere anche sino a 5 mm (Thulin, 1993). Inoltre, solo Persicaria senegalensis (nella sua f. albotomentosa) e alcune entità del gruppo di $P$. lapathifolia possono apparire lanose.

P. senegalensis è stata segnalata per la prima volta in Europa a Creta da Akeroyd (1988) con il nome di Polygonum lanigerum; questo nome è però da riferirsi alle forme lanose di P. lapathifolia (subsp. pallida) (Wilson, 1996), mentre la pianta greca, analogamente a quella di Pantelleria, è da ascrivere a $P$. senegalensis, in particolare alla forma albotomentosa. Le entità del gruppo di P. lapathifolia sono facilmente riconoscibili da tutte le altre specie del genere Persicaria per i tepali esterni provvisti di una nervatura evidente e biforcata ad àncora all'apice (cfr. Hinds 


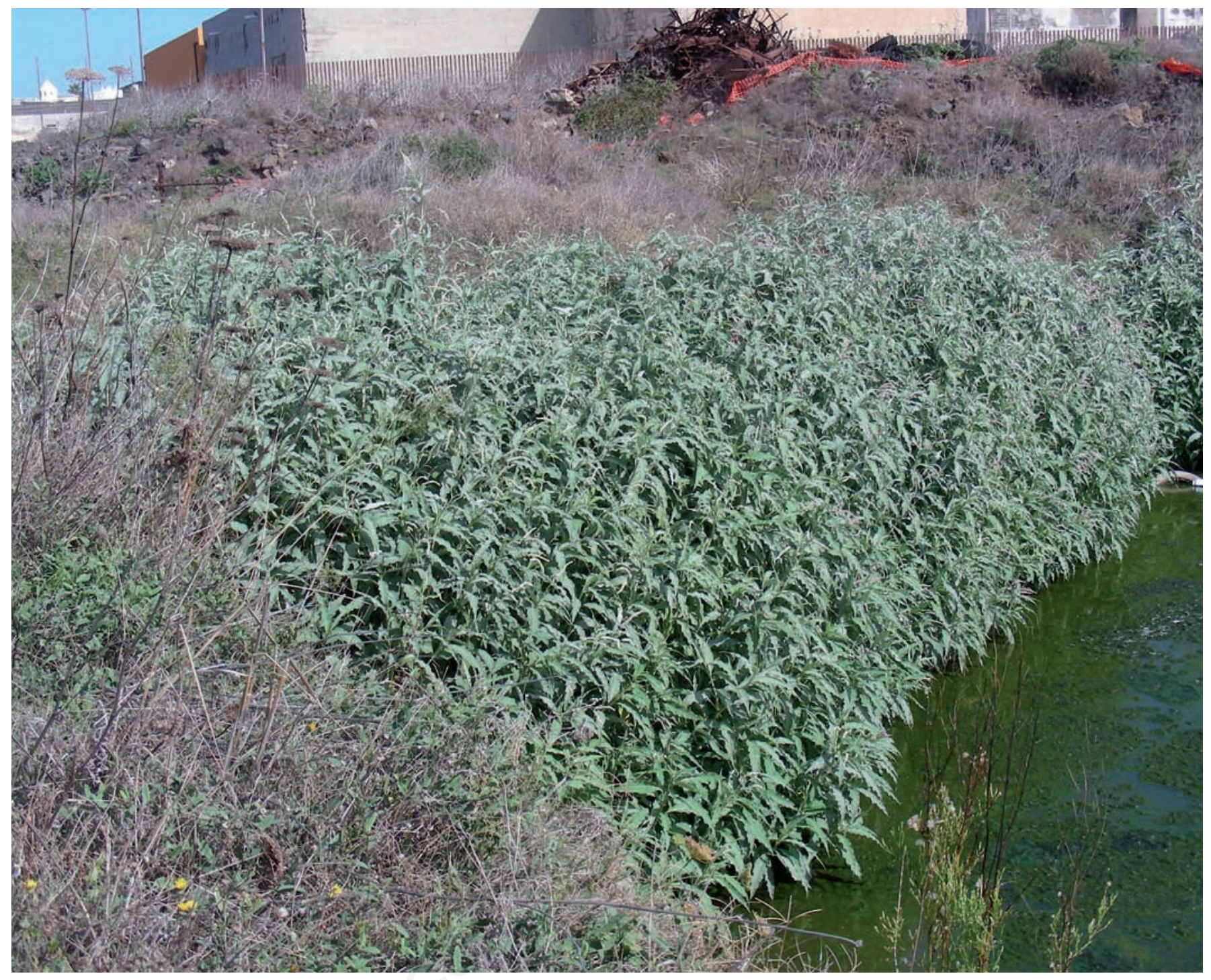

Fig. 1 - Sponda della vasca con Persicaria senegalensis. / Pool bank with Persicaria senegalensis. (Foto / Photo E. Montoleone).

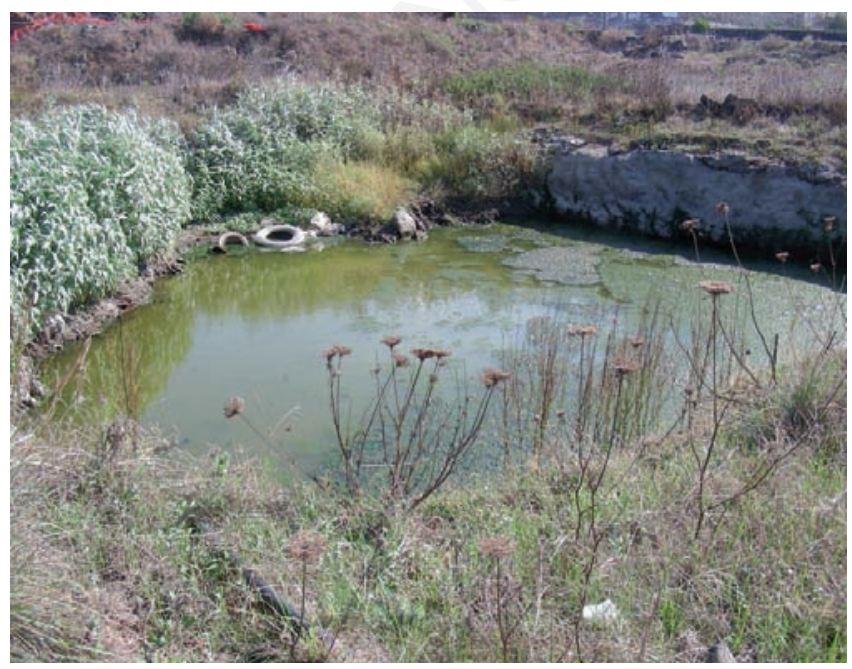

Fig. 2 - Vasca con Persicaria senegalensis e rifiuti abbandonati. / Pool bank with Persicaria senegalensis and thrown garbage (Foto / Photo E. Montoleone).
\& Freeman, 2005; Galasso, 2009; Stace, 2010), carattere facilissimo da verificare, anche a basso ingrandimento, non riportato nella maggior parte delle Flore.

Di seguito forniamo la descrizione $P$. senegalensis e una chiave dicotomica per l'identificazione di tutte le specie di Persicaria spontanee in Italia.

Persicaria senegalensis (Meisn.) Soják, Preslia, 46 (2): 155. 1974.

$\equiv$ Polygonum senegalense Meisn., Monogr. Polyg.: 54. 1826.

Lectotypus: Senegal, 12 [?] Sep 1824, Perrottet 31 (GDC, image G00138479!).

= Polygonum senegalense Meisn. f. albotomentosum R.A.Graham, Kew Bull., 11 (2): 258. 1956.

$\equiv$ Persicaria senegalensis (Meisn.) Soják f. albotomentosa (R.A.Graham) K.L.Wilson, Kew Bull., 45 (4): 630. 1990.

EPolygonum senegalense Meisn. subsp. albotomentosum (R.A.Graham) Germish., Bothalia, 16 (2): 233. 1986. 
Holotypus: Tanganyika: Utipa Distr., Lake Kwela, $5,200 \mathrm{ft}$., edge of the lake, in water, 19.3.1950, A. A. Bullock 2666 (K, image K000244080!).

- Polygonum lanigerum auct., non R.Br.

$\equiv$ Persicaria lanigera auct., non (R.Br.) Soják

三Polygonum lapathifolium L. var. lanigerum auct., non (R.Br.) C.Moore \& Betche

$\equiv$ Persicaria lapathifolia (L.) Delarbre var. lanigera auct., non (R.Br.) Chantar. \& Tubtimtong

Descrizione

Pianta perenne, robusta, rizomatosa, da glabra (nella cosiddetta f. senegalensis) a densamente bianco-lanosa (nella cosiddetta f. albotomentosa). Fusto eretto, alto fino a $3 \mathrm{~m}$, robusto, semplice, da glabro a fortemente biancotomentoso, a volte radicante dai nodi inferiori. Picciolo lungo $1-5 \mathrm{~cm}$; ocrea tubulare, lunga fino a $3.5 \mathrm{~cm}$, membranacea, bruno-rossastra, da glabra a variamente pubescente, all'apice troncata e priva di fimbrie o con deboli fimbrie lunghe fino a $5 \mathrm{~mm}$ (soprattutto nelle piante lanose); lamina da oblungo-lanceolata a lanceolata, di $8-27.5 \times 3-8 \mathrm{~cm}$, da glabra (tranne che lungo la nervatura mediana e i margini) a densamente bianco-lanosa su entrambe le facce (spesso maggiormente di sotto), inferiormente con numerose e piccole ghiandole sessili giallastre (seminascoste dal tomento nella f. albotomentosa); base cuneata e ristretta nel picciolo, apice acuto o lungamente acuminato. Infiorescenza terminale, costituita da più racemi (raramente 1) spiciformi, densi, eretti o leggermente penduli, disposti in pannocchia afilla; peduncoli lunghi fino a $7 \mathrm{~cm}$, con ghiandole arancioni, glabri o con pelosità appressata; ocreole cupoliformi, largamente ovate, troncate o arrotondate all'apice, glabre o pelose, con ghiandole arancioni, ciascuna con 2-3 fiori; pedicelli fino a 2-3 $\mathrm{mm}$, articolati presso $\mathrm{l}^{\prime}$ apice. Perianzio rosato, verdastro o bianchiccio, tetra(-penta)mero, cosparso di alcune ghiandole arancioni; tepali ellittici, lunghi 3-4 $\mathrm{mm}$, saldati alla base, con lobi di 2-2.5 mm; stami usualmente 7, dilatati alla base; stili 2, lunghi $1.5 \mathrm{~mm}$, liberi fin sotto la metà; stimmi capitati. Perianzio persistente, acheni lenticolari, con diametro di $2.5-3.5 \mathrm{~mm}$ e becco apicale di $0.5 \mathrm{~mm}$, biconcavi, bruni, lisci e lucidi (Figg. 1, 3-6).

Distribuzione

Africa, Madagascar, Medio Oriente (Israele) e Penisola Arabica (Yemen), naturalizzata in Grecia (Creta) e in Italia (Sicilia a Pantelleria). Nel continente asiatico e nelle isole del Pacifico è vicariata da P. glabra (Willd.) M.Gómez, presente anche nella Penisola Arabica.

Habitat

Rive di stagni e laghi, wadi (in Medio Oriente). Specimina visa

Italia: Sicilia, TP (Isola di Pantelleria), Pantelleria, loc. L'Arenella, pozza presso degli edifici militari abbandonati (coord. geogr. WGS84: $36.826928^{\circ} \mathrm{N}$ $\left.11.932977^{\circ} \mathrm{E}\right), 6 \mathrm{~m}$ s.l.m., no exp., sponda di pozza, 15 Oct 2012, E. Montoleone (MSNM, 43874, 43875, 43876, 44208).

Senegal: 12 [?] Sep 1824, Perrottet 31 (G-DC, microfiche G00138479).

Tanganyika: Utipa Distr., Lake Kwela, 5,200 ft., edge of the lake, in water, 19.3.1950, A. A. Bullock 2666 (K, image K000244080).

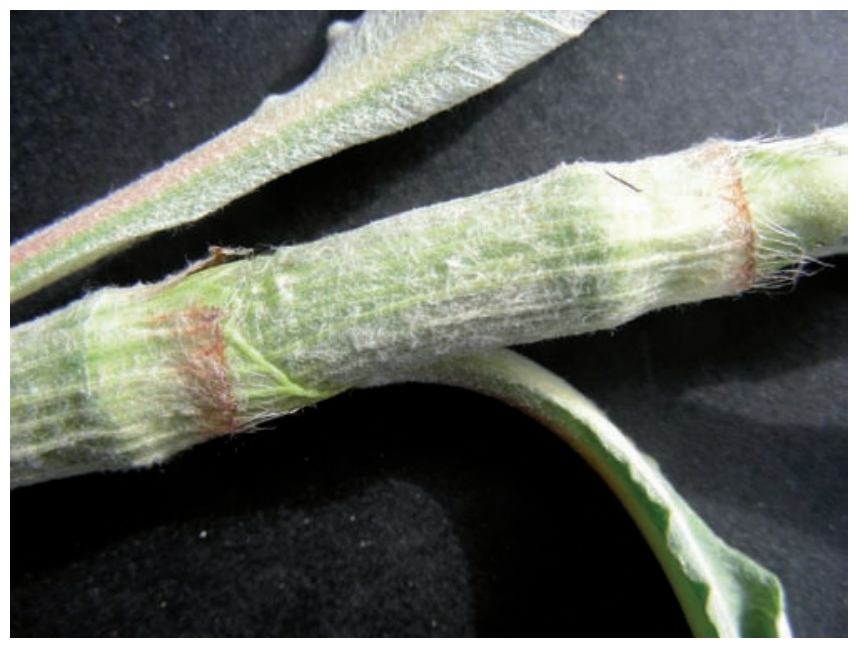

Fig. 3 - Persicaria senegalensis: ocrea, con fimbrie e superficie lanosa ocrea, with fimbriae and lanate surface. (Foto / Photo E. Montoleone).

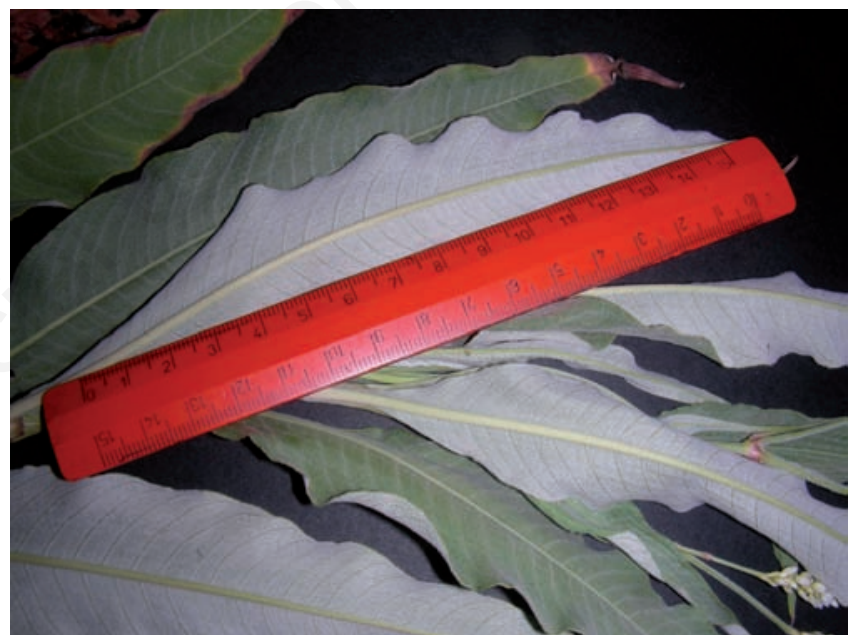

Fig. 4 - Persicaria senegalensis: foglie / leaves. (Foto / Photo E. Montoleone)

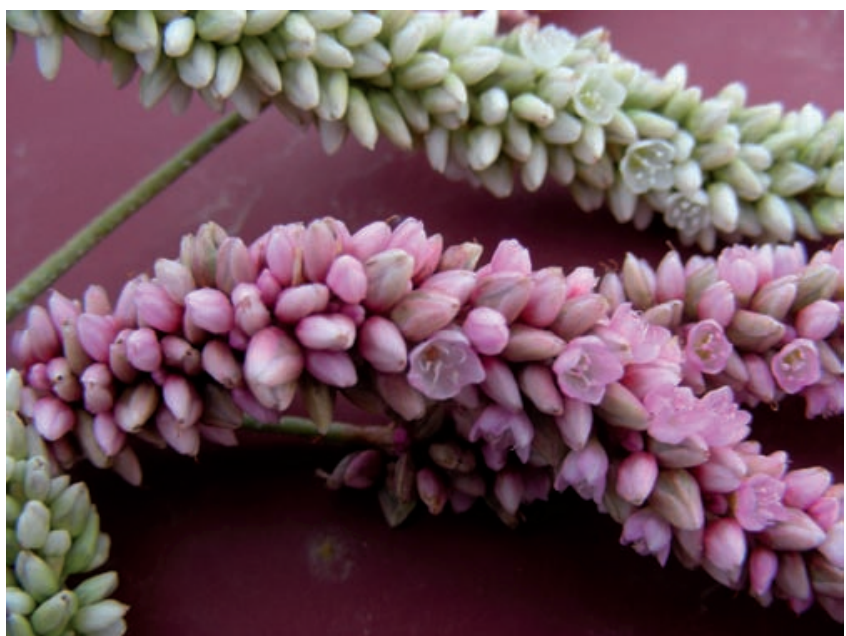

Fig. 5 - Persicaria senegalensis: fiori / flowers. (Foto / Photo E. Montoleone). 


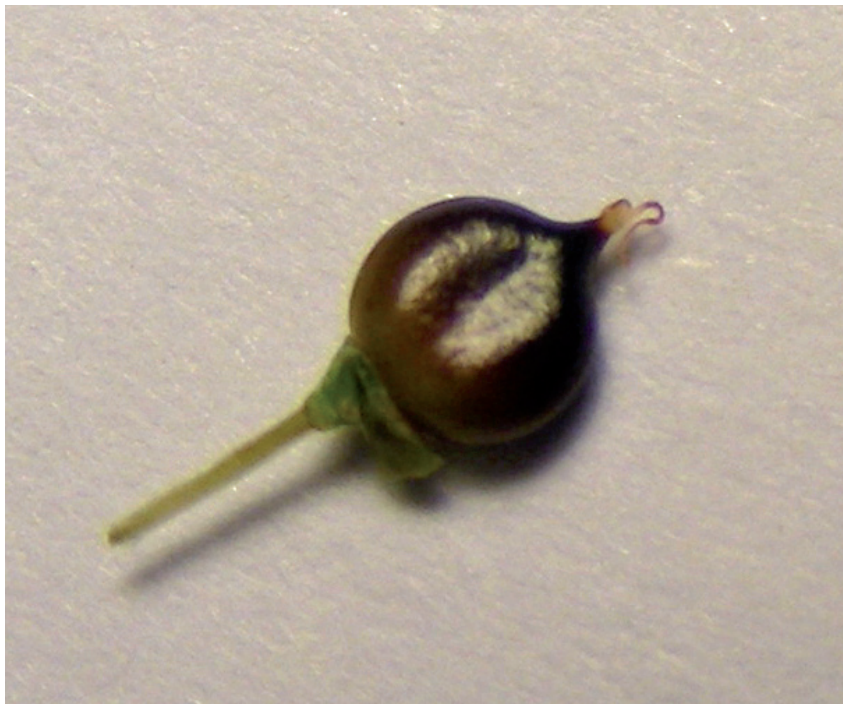

Fig. 6 - Persicaria senegalensis: frutto (achenio) / fruit (achene). (Foto ( Photo E. Montoleone).

South Africa: Limpopo Province, Farm Hoedspruit $82 \mathrm{KU}\left(24^{\circ} 26^{\prime} \mathrm{S} 31^{\circ} 00^{\prime} \mathrm{E}\right), 500 \mathrm{~m}$, aquatic - hedge of dam, 9.5.1982, N. Zambatis 1464 (BNRH, image BNRH0021096).

\section{Chiave delle specie del genere Persicaria presenti in Italia}

La presente chiave rappresenta una elaborazione e integrazione di quella pubblicata da Galasso (2009) e riporta tutte le 16 specie del genere Persicaria sinora indicate nella nostra Penisola, con l'aggiunta di 2 entità asiatiche di possibile futura comparsa: $P$. extremiorientalis e $P$. perfoliata. Si riportano anche le 5 sottospecie di P. lapathifolia (L.) Delarbre (Wißkirchen, 1995), la cui distribuzione in Italia è ancora da definire. La lettera A, posta tra parentesi posta dopo il nome del taxon. identifica le specie esotiche.

1 Fusti con spinule ricurve

2 Infiorescenze capituliformi (sect. Echinocaulon (Meisn.) H.Gross); fusti scandenti; foglie peltate e deltoidi, troncate o cordate alla base; ocree fogliacee, perfoliate e allargate attorno ai nodi; tepali carnosi e blu in frutto; achenio sferico P. perfoliata (L.) H.Gross (A) ${ }^{1}$

2 Infiorescenze spiciformi (sect. Truelloides Tzvelev); fusti eretti; foglie lanceolate o strettamente ellittiche, cuneate alla base; ocree cartacee, cilindriche e aderenti ai fusti; tepali cartacei in frutto; achenio biconvesso ....

$$
\text { P. bungeana (Turcz.) Nakai (A) }
$$

1 Fusti senza spinule

3 Infiorescenze capituliformi (sect. Cephalophilon (Meisn.) H.Gross)

4 Pianta annuale, erbacea; pagina inferiore delle foglie con ghiandole puntiformi; acheni biconvessi, opachi P. nepalensis (Meisn.) H.Gross (A)

\footnotetext{
${ }^{1}$ Non ancora segnalata in Italia.
}

4 Pianta perenne, legnosa alla base; pagina inferiore delle foglie con peli ghiandolari; acheni trigoni, lucidi ......... P. capitata (Buch.-Ham. ex D.Don) H.Gross (A)

3 Infiorescenze spiciformi

5 Infiorescenze strettamente lineari, molto lasse; stili persistenti nel frutto, induriti e ricurvi a uncino; foglie larghe, ovate od obovate(sect.Tovara (Adans.)H.Gross)

6 Foglie ovate, acute all'apice; tepali verdastri o bianchi, raramente rosa ............ P. virginiana (L.) Gaertn. (A)

6 Foglie obovate, ottuse all'apice; tepali rosa o rossastri P. filiformis (Thunb.) Nakai (A)

5 Infiorescenze non strettamente lineari, dense $\mathrm{O}$ lasse; stili non come sopra; foglie strette, lineari-lanceolate o lanceolate, ovate in $P$. orientalis

7 Pianta anfibia, perenne, rizomatosa; picciolo inserito circa a metà dell'ocrea; infiorescenze solo terminali (sect. Amphibia Tzvelev) ... P. amphibia (L.) Delarbre

7 Pianta terrestre, annuale o perenne, in quest'ultimo caso senza rizomi ma con stoloni; picciolo inserito nella metà inferiore dell'ocrea, spesso alla base; infiorescenze terminali e ascellari (sect. Persicaria)

8 Infiorescenze dense

9 Foglie ovate, larghe 3-17 cm; ocree all'apice espanse in un'ala fogliacea verde...........P. orientalis (L.) Spach (A)

9 Foglielanceolate, più strette; ocreenon espanseall'apice

10 Tepali esterni con nervatura prominente e terminante $\mathrm{ad}$ àncora (P. lapathifolia (L.) Delarbre)

11 Fusti generalmente sfumati o punteggiati di rosso (antociani), perlopiù con 14-30 o più nodi e internodi relativamente brevi; foglie verde puro, un po' arrossate all'appassimento, con macchia sulla pagina superiore spesso ben evidente, a volte assente; infiorescenze terminali lasse, generalm. composte da 4-5 spighe peduncolate, a volte disposte a pannocchia, piuttosto slanciate (diametro circa 6-8 $\mathrm{mm}$ ), spesso pendule; asse dell'infiorescenza e fiori spesso solo debolmente ghiandolari, perianzio (al termine della fioritura) biancasto o rosa, mai verde, generalmente racchiudente completamente l'achenio maturo, con nervi in rilievo ed evidentemente a forma di àncora; acheni generalmente ovati, di rado tondi, con base distintamente arrotondata, lunghi $1.8-2.3 \mathrm{~mm}$ (stilo escluso)

12 Piante generalmente ascendenti o erette, in condizioni ambientali favorevoli relativamente alte (fino a $1.5 \mathrm{~m}$ o più); foglie lanceolate, lunghe circa 3-6 volte la propria larghezza ....... P. lapathifolia subsp. lapathifolia

12 Piante generalmente prostrate o leggermente ascendenti, erette solo se molto fitte, perlopiù basse; foglie brevemente ovato-lanceolate, ovate o tondeggianti, almeno le inferiori lunghe non più del doppio della propria larghezza

P. lapathifolia subsp. brittingeri (Opiz) Soják

11 Fusti generalmente verdi, raramente con una leggera sfumatura rossiccia, perlopiù con 7-14 o meno nodi e internodi relativamente lunghi; foglie maggiormente grigio-verdi, giallastre all'appassimento, con macchia sulla pagina superiore assente o indistinta; infiorescenze terminali compatte, generalmente composte da 1-2 spighe, le laterali spesso ridotte e sessili, tozzamente coniche (diametro circa $8-10 \mathrm{~mm}$ ), perlopiù erette o leggermente arcuate; asse dell'infiorescenza 
e fiori spesso più fortemente ghiandolari, perianzio (al termine della fioritura) distintamente verdastro, di rado con sfumature rossastre, spesso più breve dell'achenio maturo, con nervi almeno in alcuni casi non sporgenti e/o a forma di àncora irregolare; acheni tondi, con base da leggermente arrotondata a troncata, lunghi perlopiù 2.3-3 $\mathrm{mm}$ (stilo escluso)[piante con caratteristiche intermedie tra $\mathrm{i}$ due punti 11 sono state indicate da Wißkirchen (1995) come subsp. mesomorpha (Danser) Soják]

13 Internodi non straordinariamente lunghi e slanciati, ramificazioni patenti o eretto-patenti; foglie largamente lanceolate; frutti maturi caduchi (pedicello articolato); nervi del perianzio almeno in parte soporgenti . P. lapathifolia subsp. pallida (With.) Á.Löve

13 Internodi straordinariamente lunghi e slanciati, ramificazioni erette, spesso più lunghe del fusto principale oppure piante non ramificate; foglie strettamente lanceolate; frutti maturi non caduchi (pedicello perlopiù non articolato), con perianzio spesso distintamente più corto dell'achenio; nervi del perianzio non sporgenti e perlopiù anastomizzati

........ P. lapathifolia subsp. leptoclada (Danser) Wissk.

10 Tepali esterni non come sopra

14 Fusti sotto l'infiorescenza e peduncoli con ghiandole stipitate ....... P. pensylvanica (L.) M.Gómez (A)

14 Fusti sotto l'infiorescenza e peduncoli glabri o con ghiandole sessili o subsessili (stipite $<$ ghiandola)

15 Pianta perenne, spesso bianco-lanosa; pagina inferiore delle foglie con ghiandole puntiformi, la superiore senza macchia nera a V rovesciata; ocree senza fimbrie o con fimbrie brevi rispetto alla lunghezza dell'ocrea stessa (anche se fino a $4-5 \mathrm{~mm}$ ) P. senegalensis (Meisn.) Soják (A)

15 Pianta annuale, sempre verde; pagina inferiore delle foglie senza ghiandole puntiformi, la superiore spesso con macchia nera a $\mathrm{V}$ rovesciata; ocree con fimbrie evidenti rispetto alla lunghezza dell'ocrea stessa

16 Fusti glabri; setole al margine delle foglie lunghe 0.2-0.5 mm; peduncoli raramente stipitato-ghiandolari (stipite $<$ ghiandola); spighe lunghe $1-4 \mathrm{~cm}$, erette; tepali in frutto con nervature prominenti nel secco; acheni non tumescenti alla base .....

P. maculosa Gray

16 Fusti generalmente pelosi; setole al margine delle foglie lunghe 0.5-1.2 $\mathrm{mm}$; peduncoli generalmente stipitato-ghiandolari (stipite $<$ ghiandola), raramente con ghiandole minute o pressoché assenti; spighe usualmente lunghe $3-8 \mathrm{~cm}$, di solito ricurve; tepali in frutto con nervature non prominenti nel secco; acheni tumescenti alla base

P. extremiorientalis (Vorosch.) Tzvelev (A) ${ }^{2}$

8 Infiorescenze lasse e interrotte

17 Tepali densamente punteggiati di ghiandole traslucide; foglie con sapore acre di pepe .....

P. hydropiper (L.) Delarbre

17 Tepali senza ghiandole o queste molto scarse; foglie con sapore erbaceo

\footnotetext{
${ }^{2}$ Non ancora segnalata in Italia.
}

18 Pianta perenne, con lunghi stoloni o fusti decombenti e radicanti ai nodi; foglie maggiori più lunghe di 10 $\mathrm{cm}$ P. decipiens (R.Br.) K.L.Wilson

18 Pianta annuale; foglie maggiori raramente più lunghe di $8 \mathrm{~cm}$

19 Pianta densamente cespitosa e ramificata, con lunghi rami prostrati e radicanti ai nodi basali

P. longiseta (Bruijn) Kitag. (A)

19 Pianta non densamente cespitosa, senza rami lungamente prostrati e radicanti ai nodi, eretta o erettoascendente

20 Foglie inferiori meno di 5 volte più lunghe che larghe, larghe 12-30 mm; acheni lunghi 2.5-4 mm, biconvessi o subtrigoni

P. dubia (Stein) Fourr.

20 Foglie inferiori più di 5 volte più lunghe che larghe, larghe 2-15 mm; acheni lunghi 1.8-2.5 mm, biconvessi P. minor (Huds.) Opiz

\section{Ringraziamenti} e Kew.

Gli autori ringraziano i curatori degli erbari di Ginevra

\section{BIBLIOGRAFIA}

Akeroyd J. R., 1988 - Two overlooked species of Polygonum from SE Europe. In: Flora Europaea: Notulae systematicae ad Floram Europaeam spectantes. Series 2. No. 1. Chater A. O. (ed.). Botanical Journal of the Linnean Society, London, 95 (4): 251-257.

Atha D. E., Nee M. H. \& Naczi R. F. C., 2010 - Persicaria extremiorientalis (Polygonaceae) is extablished in the flora of the eastern United States of America. Journal of the Torrey Botanical Society, Lawrence, 137 (4): 333-338.

Boulos L., 1999 - Flora of Egypt. Al Hadara Publishing, Cairo, 1.

Brusa G. \& Galasso G., 2006 - Notula: 1266. In: Notulae alla checklist della flora vascolare italiana: 2 (1192-1266). Conti F., Nepi C. \& Scoppola A. (eds.). Informatore Botanico Italiano, Firenze, 38 (1): 214 .

Conti F., Abbate G., Alessandrini A. \& Blasi C. (eds.), 2005 - An Annotated Checklist of the Italian Vascular Flora. Palombi Editori, Roma.

El Husseini N. \& El Hadidi M. N., 2000 - 19. Polygonaceae Juss. In: Flora Aegyptiaca. El Hadidi M. N. (ed.). The Palm Press, Cairo, 1 (2): 64-82.

Galasso G., 2007 - Notula: 1332. In: Notulae alla checklist della flora vascolare italiana: 4 (1311-1419). Conti F., Nepi C., Peruzzi L. \& Scoppola A. (eds.). Informatore Botanico Italiano, Firenze, 39 (2): 409.

Galasso G., 2009 - I poligoni (Polygonum s.1., Polygonaceae) italiani: ridelimitazione dei generi e proposte per una chiave. In: Gruppi critici della Flora d'Italia, Comunicazioni. Dipartimento di Biologia Vegetale, La Sapienza Università di Roma, 30-31 ottobre 2009. Peccenini S. \& Domina G. (eds.). Società Botanica Italiana, Firenze: 47-49. 
Galasso G., Banfi E., De Mattia F., Grassi F., Sgorbati S. \& Labra M., 2009 - Molecular phylogeny of Polygonum L. s.l. (Polygonoideae, Polygonaceae), focusing on European taxa: preliminary results and systematic considerations based on $r b c \mathrm{~L}$ plastidial sequence data. Atti Società italiana di Scienze naturali e del Museo civico di Storia naturale in Milano, Milano, 150 (1): 113-148.

Galasso G. \& Brusa G., 2007 - Notula: 1281. In: Notulae alla checklist della flora vascolare italiana: 3 (1267-1310). Conti F., Nepi C. \& Scoppola A. (eds.). Informatore Botanico Italiano, Firenze, 39 (1): 241242.

Galasso G., Mauri S. \& Cattaneo G., 2006 - Notula: 1264. In: Notulae alla checklist della flora vascolare italiana: 2 (1192-1266). Conti F., Nepi C. \& Scoppola A. (eds.). Informatore Botanico Italiano, Firenze, 38 (1): 213.

Galasso G. \& Tomasi D., 2007 - Notula: 1331. In: Notulae alla checklist della flora vascolare italiana: 4 (1311-1419). Conti F., Nepi C., Peruzzi L. \& Scoppola A. (eds.). Informatore Botanico Italiano, Firenze, 39 (2): 408-409.

Graham R. A., 1958 - Polygonaceae. In: Flora of Tropical East Africa. Turrill W. B. \& Milne-Redhead E. (eds.). Whitefriars Press Ltd., London, Tornbridge.

Hinds H. R. \& Freeman C. C., 2005 - 32. Persicaria (Linnaeus) Miller. In: Flora of North America North of Mexico. Flora of North America Editorial Committee (ed.). Oxford University Press, New York, Oxford, 5 (Magnoliophyta: Caryophyllidae, part 2): 574-594.
Nyberg J. A. \& Miller A. G., 1996 - Family 32. Polygonaceae. In: Flora of the Arabian Peninsula and Socotra. Miller A. G. \& Cope T. A. (eds). Edinburgh University Press, Edinburgh, 1: 127-143.

Pignatti S., 1982 - Flora d'Italia. Edagricole, Bologna, 1.

Poindexter D. B., 2010 - Persicaria perfoliata (Polygonaceae) reaches North Carolina. Phytoneuron, Fort Worth, 2010 (30): 1-9.

Stace C. A., 2010 - New flora of the British Isles. $3^{\text {rd }}$ ed. Cambridge University Press, Cambridge.

Thulin M., 1993 - 42. Polygonaceae. In: Flora of Somalia. Thulin M. (ed.). Royal Botanic Gardens, Kew, 1: 175-179.

Wilson K. L., 1990 - Some widespread species of Persicaria (Polygonaceae) and their allies. Kew Bulletin, London, 45 (4): 621-636.

Wilson K. L., 1996 - Nomenclatural notes on Polygonaceae in Australia. Telopea, Sidney, 7 (1): 83-94.

Wißkirchen R., 1995 - Zur Bestimmung der Unterarten von Polygonum lapathifolium L. s.l. Floristische Rundbriefe, Bochum, 29 (1): 1-25.

Zohary M., 1966 - Flora Palaestina. The Israel Academy of Sciences and Humanities, Jerusalem. 